\title{
Telling in-tune from out-of-tune: widespread evidence for implicit absolute intonation
}

\author{
Stephen C. Van Hedger ${ }^{1} \cdot$ Shannon L. M. Heald $^{1}$ - Alex Huang ${ }^{1}$ - Brooke Rutstein ${ }^{1}$ \\ Howard C. Nusbaum ${ }^{1}$
}

Published online: 6 July 2016

(C) Psychonomic Society, Inc. 2016

\begin{abstract}
Absolute pitch (AP) is the rare ability to name or produce an isolated musical note without the aid of a reference note. One skill thought to be unique to AP possessors is the ability to provide absolute intonation judgments (e.g., classifying an isolated note as "in-tune" or "out-of-tune"). Recent work has suggested that absolute intonation perception among AP possessors is not crystallized in a critical period of development, but is dynamically maintained by the listening environment, in which the vast majority of Western music is tuned to a specific cultural standard. Given that all listeners of Western music are constantly exposed to this specific cultural tuning standard, our experiments address whether absolute intonation perception extends beyond AP possessors. We demonstrate that non-AP listeners are able to accurately judge the intonation of completely isolated notes. Both musicians and nonmusicians showed evidence for absolute intonation recognition when listening to familiar timbres (piano and violin). When testing unfamiliar timbres (triangle and inverted sine waves), only musicians showed weak evidence of absolute intonation recognition (Experiment 2). Overall, these results highlight a previously unknown similarity between AP and non-AP possessors' long-term musical note representations, including evidence of sensitivity to frequency.
\end{abstract}

Electronic supplementary material The online version of this article (doi:10.3758/s13423-016-1099-1) contains supplementary material, which is available to authorized users.

Stephen C. Van Hedger

shedger@uchicago.edu

1 Department of Psychology, The University of Chicago, 5848 S. University Ave., Chicago, IL 60637, USA
Keywords Implicit learning and memory $\cdot$ Music cognition · Sound recognition $\cdot$ Categorization $\cdot$ Perceptual implicit memory

To what extent do we retain the absolute features of our listening environment? In music, this question is often situated within the phenomenon of absolute pitch (AP)-the ability to name or produce an isolated musical note with no reference point (see Deutsch, 2013, for a review). In addition to providing category labels for isolated notes, AP possessors also can provide absolute intonation judgments for musical notes - that is, determine whether an isolated note is "in-tune" or "out-of-tune" (e.g., Miyazaki, 1988). Although these judgments are not "perfect" among AP possessors (e.g., Lockhead \& Byrd, 1981), they are definitionally above chance performance levels. Both abilities are thought to be crystallized during a critical period of development and remain stable throughout development (cf. Ward \& Burns, 1982). However, recent evidence has suggested that intonation judgments among AP possessors are malleable and dependent on one's environment. Specifically, listening to a flattened symphony can temporarily shift an AP possessor's sense of what is "intune," suggesting that absolute intonation is at least in part held in place by the listening environment (Hedger, Heald, \& Nusbaum, 2013).

The fact that the listening environment influences an AP possessor's sense of intonation suggests that the statistical regularities of our listening environments play an important role in absolute intonation perception (cf. Saffran, Johnson, Aslin, \& Newport, 1999). Given that statistical learning is often described as an implicit mechanism for acquiring information from our auditory environments, it is possible that all individuals - regardless of an ability to explicitly label or produce a musical note name-might possess some absolute pitch 
information that is tied to the regularities encountered in the environment. Indeed, the modal response for individuals humming familiar recording melodies from memory matches the key signature found in the recording (Jakubowski \& Müllensiefen, 2013; Levitin, 1994). Additionally, individuals can perceive when they are hearing a version of a familiar recording that has been slightly shifted in pitch (e.g., Schellenberg \& Trehub, 2003).

To what extent does this pitch knowledge, sometimes referred to as implicit absolute pitch, generalize beyond the specific instances of well-known recordings? Recently, Ben-Haim, Eitan, and Chajut (2014) found that non-AP possessors rated isolated notes as more pleasing if they occurred less frequently in the environment. While Ben-Haim et al. (2014) provide some evidence that note categories are represented in long-term memory, the nature of these representations remains unclear. This is because a post hoc explanation is needed to interpret the relationship between pleasantness and frequency of occurrence, and "pleasantness" is not typically tied to note identity. In this article we use a novel approach, situated within previous categorization literature, to understand the nature of non-AP possessors' absolute pitch representations. One of the more robust findings in categorization research is the notion of typicality, or the idea that some category members are more prototypical than others (Rosch, 1973). Taking advantage of this principle, our experiments examine whether non-AP possessors' have distinct absolute pitch representations that include typicality, which would confer the ability to accurately differentiate intune from out-of-tune notes - previously thought to be exclusive to AP possessors. For example, Miyazaki (1988) found that non-AP possessors could not distinguish between in-tune and out-of-tune notes, though the experimental paradigm required explicit note labeling in addition to intonation judgments. As such, it is difficult to ascertain whether non-AP possessors can differentiate in-tune from out-of-tune notes in a task that does not require the explicit labeling of a note.

Given that typicality is a property of a category, how could non-AP possessors show such effects in the absence of having explicit note categories? One possibility is that non-AP listeners may have formed implicit note categories. There is substantial stability of intonation in the listening environment, because the vast majority of Western music is tuned to a specific standard. Although adjacent musical notes are typically separated by exactly 100 cents (one semitone) in Western music, the system is often absolutely fixed such that the " $\mathrm{A}$ " above "middle $C$ " is tuned to $440 \mathrm{~Hz}$, hereafter referred to as canonical tuning. This could form the basis for implicit note categories based on the structure of listening experience. If everyday listeners possess note representations based on canonical tuning, then they might be able to identify when an isolated note either conforms to this standard or is 50 cents removed, because 50 cents represents the maximal allowable deviations between two adjacent notes. On the other hand, the ability to make absolute intonation judgments may only manifest if one possesses AP.

\section{Experiment 1}

\section{Method}

\section{Participants}

One hundred five participants were recruited through Amazon Mechanical Turk (MTurk). ${ }^{1}$ Participants had to be residing in the United States as well as have a minimum $90 \%$ satisfactory completion rate from prior MTurk assignments. Three participants were excluded from all analyses because they reported possessing AP, leaving 102 analyzable participants.

\section{Materials}

We created 48 musical notes (1,000 ms in duration) with Reason music production software (Propellerhead; Stockholm, Sweden). Half of the notes had a piano timbre, while the remaining half had a violin timbre. Within each instrumental category, participants heard exactly 12 in-tune notes (corresponding to canonical tuning) and 12 out-of-tune notes. The out-of-tune notes were shifted up in pitch by 50 cents, meaning that the out-of-tune notes fell exactly between two canonically tuned notes (see Fig. 1). The 24 notes within each instrument category spanned a one-octave range (A $220.00 \mathrm{~Hz}$ to $\mathrm{G \#}{ }^{+50} 427.47 \mathrm{~Hz}$ ). Because Reason uses MIDI information, we were able to shift the pitch of the out-of-tune notes prior to exporting them as audio files. A 10second masking sound, presented between trials to minimize carryover effects, was created in Adobe Audition (Adobe Systems; San Jose, CA) and consisted of both white noise and a continuous pitch sweep from $0 \mathrm{~Hz}$ to $760 \mathrm{~Hz}$ and back to $0 \mathrm{~Hz}$. All sounds were root mean square normalized to -13 dB. The experiment was coded in jsPsych (de Leeuw, 2014).

\section{Procedure}

After providing consent, participants heard a 10-second white noise sample and were instructed to adjust their volume to a level at which the noise was being played at a comfortable volume. Then participants were instructed that they would hear several isolated musical notes, with some being "in-tune"

\footnotetext{
${ }^{1}$ The sample size for all experiments was determined by availability of funds in addition to a prospective power analysis, in which achieving a power of 0.8 would require a minimum of 88 participants to detect a difference of 3 percentage points above chance (with an estimated standard deviation of 10 percentage points).
} 


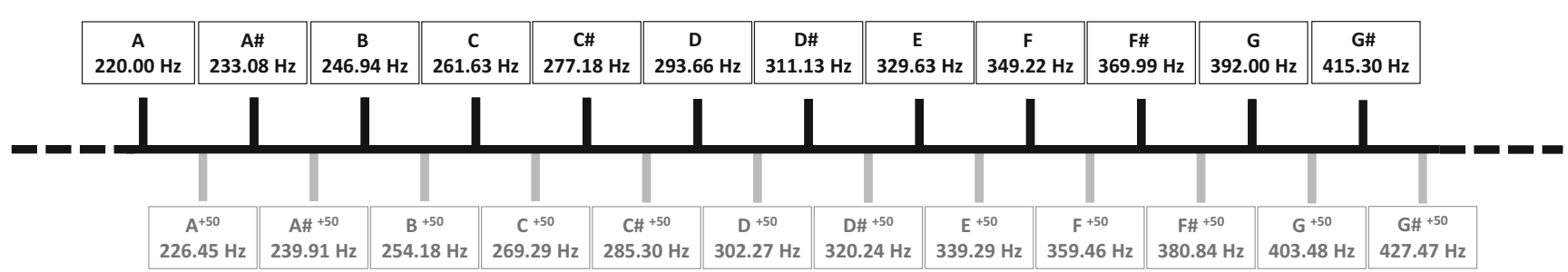

Fig. 1 Frequencies tested across both experiments. The notes represented in black are canonically tuned, whereas the notes represented in gray fall exactly in between canonically tuned notes (offset by approximately $2.93 \%$, or 50 cents)

and others being "out-of-tune." Given that we did not specifically recruit musicians for this task, we wanted to make sure that participants understood what was meant by these terms. We explained in the instructions that most Western music is tuned to a specific standard, and that some of the notes they would hear would conform to this standard whereas other notes they would hear would not conform to this standard.

Table 1 Experiment 1 and $1 \mathrm{~B}$ intonation accuracy and statistical analyses. Accuracy is represented in terms of both the proportion correct and the number of total correct trials. We analyzed the data through a one-sample $t$ test as well as through a Bayesian equivalent of a
We additionally defined the category distinction as how "good" or "bad" a note sounds, where "good" was defined as typical by canonical tuning standards.

Participants heard all 48 notes in a random order. Before hearing each note, participants were given a 1,500-ms visual countdown (in which ***, **, and * were each sequentially presented for $500 \mathrm{~ms}$ ) to ensure that participants were

one-sample $t$ test (represented through the Bayes factor, $\mathrm{BF}_{10}$ ). The final column represents the $95 \%$ confidence interval of the standardized effect size

\begin{tabular}{|c|c|c|c|c|c|c|}
\hline \multicolumn{7}{|c|}{ Experiment 1} \\
\hline \multicolumn{7}{|c|}{ All participants $(n=102)$} \\
\hline Timbre & $\begin{array}{l}\text { Mean Proportion } \\
\text { Correct }\end{array}$ & $\begin{array}{l}\text { Mean Number } \\
\text { Correct }\end{array}$ & $t(d f)$ & $p$ value & $\mathrm{BF}_{10}$ & $95 \% \mathrm{CI}(\delta)$ \\
\hline Piano & 0.567 & $13.61 / 24$ & $6.80(101)$ & $<0.001$ & $1.33 \mathrm{E} 7$ & {$[0.42,0.84]$} \\
\hline Violin & 0.549 & $13.18 / 24$ & $4.26(101)$ & $<0.001$ & 371 & {$[0.21,0.61]$} \\
\hline Overall & 0.558 & $26.78 / 48$ & $6.52(101)$ & $<0.001$ & $3.76 \mathrm{E} 6$ & {$[0.44,0.88]$} \\
\hline \multicolumn{7}{|c|}{ Musicians $(n=48)$} \\
\hline Timbre & $\begin{array}{l}\text { Mean Proportion } \\
\text { Correct }\end{array}$ & Mean Number Correct & $t(d f)$ & $p$ value & $\mathrm{BF}_{10}$ & $95 \%$ CI $(\delta)$ \\
\hline Piano & 0.609 & $14.60 / 24$ & $7.30(47)$ & $<0.001$ & $4.06 \mathrm{E} 6$ & {$[0.67,1.37]$} \\
\hline Violin & 0.575 & $13.79 / 24$ & $4.05(47)$ & $<0.001$ & 128 & {$[0.26,0.86]$} \\
\hline Overall & 0.592 & $28.40 / 48$ & $6.31(47)$ & $<0.001$ & $1.58 \mathrm{E} 5$ & {$[0.54,1.22]$} \\
\hline \multicolumn{7}{|c|}{ Nonmusicians $(n=54)$} \\
\hline Timbre & $\begin{array}{l}\text { Mean Proportion } \\
\text { Correct }\end{array}$ & $\begin{array}{l}\text { Mean Number } \\
\text { Correct }\end{array}$ & $t(d f)$ & $p$ value & $\mathrm{BF}_{10}$ & $95 \%$ CI $(\delta)$ \\
\hline Piano & 0.529 & $12.70 / 24$ & $2.74(53)$ & 0.008 & 4 & {$[0.08,0.63]$} \\
\hline Violin & 0.526 & $12.61 / 24$ & $1.90(53)$ & 0.063 & 1 & {$[-0.02,0.51]$} \\
\hline Overall & 0.527 & $25.32 / 24$ & $3.10(53)$ & 0.003 & 10 & {$[0.12,0.68]$} \\
\hline \multicolumn{7}{|c|}{ Musicians vs. Nonmusicians (Difference Score) } \\
\hline Timbre & $\begin{array}{l}\text { Mean Proportion } \\
\text { Correct }\end{array}$ & $\begin{array}{l}\text { Mean Number } \\
\text { Correct }\end{array}$ & $t(d f)$ & $p$ value & $\mathrm{BF}_{10}$ & $95 \%$ CI $(\delta)$ \\
\hline Piano & 0.079 & $1.90 / 24$ & $4.39(100)$ & $<0.001$ & 675 & {$[0.42,1.22]$} \\
\hline Violin & 0.049 & $1.18 / 24$ & $2.19(100)$ & 0.031 & 2 & {$[0.02,0.78]$} \\
\hline Overall & 0.064 & $3.08 / 48$ & $3.78(78)^{*}$ & $<0.001$ & 123 & {$[0.33,1.12]$} \\
\hline \multicolumn{7}{|c|}{ Experiment 1B } \\
\hline \multicolumn{7}{|c|}{ All participants $(n=95)$} \\
\hline Timbre & $\begin{array}{l}\text { Mean Proportion } \\
\text { Correct }\end{array}$ & Mean Number Correct & $t(d f)$ & $p$ value & $\mathrm{BF}_{10}$ & $95 \% \mathrm{CI}(\delta)$ \\
\hline Piano & 0.568 & $13.64 / 24$ & $6.21(94)$ & $<0.001$ & $8.05 \mathrm{E} 5$ & {$[0.40,0.84]$} \\
\hline Violin & 0.532 & $12.76 / 24$ & $3.15(94)$ & 0.002 & 11 & {$[0.11,0.52]$} \\
\hline Overall & 0.550 & $26.40 / 48$ & $6.25(95)$ & $<0.001$ & $9.26 \mathrm{E} 5$ & {$[0.40,0.84]$} \\
\hline
\end{tabular}

* Equal variances were not assumed, and thus the degrees of freedom were adjusted accordingly 
prepared to hear the note. After hearing each note, participants made a forced-choice judgment regarding absolute intonation ("in-tune" or "out-of-tune"). After this judgment, participants heard the 10-second mask, which minimized the chances of an echoic trace of the previous trial from influencing the judgment on the next trial (e.g., Darwin, Turvey, \& Crowder, 1972). At the end of the experiment, participants were asked about their musical experience-specifically, whether they had ever played a musical instrument (used to bifurcate musicians and nonmusicians), their primary instrument (if applicable), years of explicit training (if applicable), whether they were actively playing music (if applicable), age of beginning musical instruction (if applicable), and whether they possessed absolute pitch. Table S1 in the Supplementary Material summarizes these musical experience results.

\section{Results}

\section{Overall Intonation Accuracy}

We first assessed whether all participants showed evidence of absolute intonation, operationalized as accuracy that was significantly greater than $50 \%$ correct (chance performance). We assessed performance using both nullhypothesis significance testing (NHST) and Bayes factors $\left(\mathrm{BF}_{10}\right)$ using JASP 0.7.5.6 (JASP Team, 2016). The $\mathrm{BF}_{10}$ assesses how much more likely the data are to have occurred under the alternative hypothesis $\left(\mathrm{H}_{1}\right)$ compared to the null hypothesis $\left(\mathrm{H}_{0}\right)$ given the priors assumed in the model. Collapsed across all 102 analyzable participants, we found considerable evidence for our hypothesis that participants could differentiate canonically tuned from noncanonically tuned notes. Overall, the proportion of trials in which participants chose the correct intonation label was 0.558 . These results, while modestly above chance, were consistent across individuals and provided strong evidence for the effect using both NHST and $\mathrm{BF}_{10}{ }^{2}$ Table 1 provides a summary of the NHST and $\mathrm{BF}_{10}$ analyses. These results are particularly notable because the notes were presented in isolation (i.e., outside of a relative pitch or even musical context).

\section{Musical Experience Differences}

Given the near even split of self-identified musicians $(n=48)$ and nonmusicians $(n=54)$ in our sample, we assessed whether

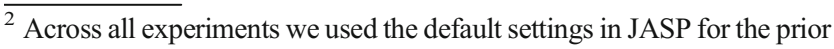
distribution on effect sizes - that is, a Cauchy prior with scale parameter, $r=0.707$.
}

musical training resulted in a better sense of absolute intonation. The proportion of trials in which musicians chose the correct intonation category was 0.592 , and the proportion of trials in which nonmusicians chose the correct intonation category was 0.527 . This difference was significant using NHST and provided decisive evidence in favor of musicians outperforming nonmusicians as assessed through the $\mathrm{BF}_{10}$ (see Table 1). However, both musicians and nonmusicians showed moderate evidence for above-chance performance, at least when collapsing performance across timbre. Figure $2 \mathrm{a}$ shows a histogram of overall intonation accuracy across both musicians and nonmusicians.

\section{Experiment 1B}

To confirm that the findings from Experiment 1 could not be attributed to confounding factors, such as participants checking their answers against canonically tuned music in another Web browser, Experiment 1B aimed to replicate the effects of Experiment 1 inside a controlled laboratory setting. These data were collected as a part of a follow-up experiment in which participants similarly rated the intonation of isolated piano and violin timbres.

\section{Method}

\section{Participants}

We recruited 101 University of Chicago undergraduates to participate. The majority of participants (92) reported at least some musical instruction. Of the 101 participants, six were excluded from all analyses because they reported possessing AP. This left 95 analyzable participants.

\section{Materials and Procedure}

The musical notes were identical to those used in Experiment 1. The procedure was virtually identical, with the exception that the trials were blocked by timbre (piano followed by violin) rather than random. This difference was related to the primary experimental question of the follow-up study and did not appear to substantially affect performance (see Table 1). The experiment was run using E-Prime (Psychology Software Tools; Sharpsburg, PA). Additionally, we normalized the volume prior to each experimental session (to approximately $70 \mathrm{~dB}$ SPL), obviating the need for the initial volume test that was used in Experiment 1. 


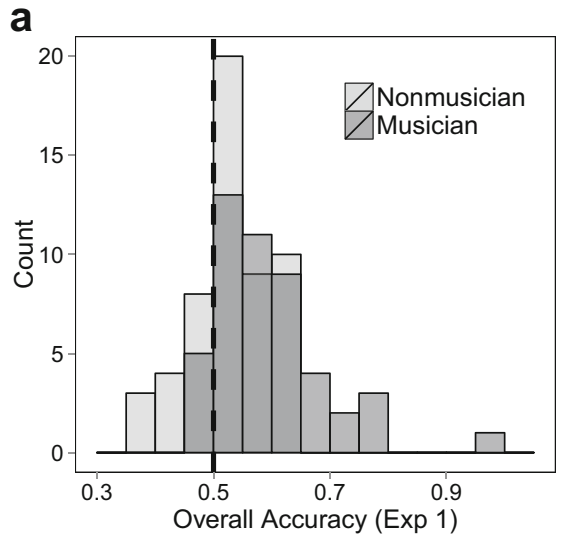

Fig. 2 Histograms of overall intonation accuracy across musicians and nonmusicians for Experiments 1 (a), 1B (b), and 2 (c). Removing the highly accurate performers $(x>0.8)$ from Experiments 1 and $1 \mathrm{~B}$ did not b

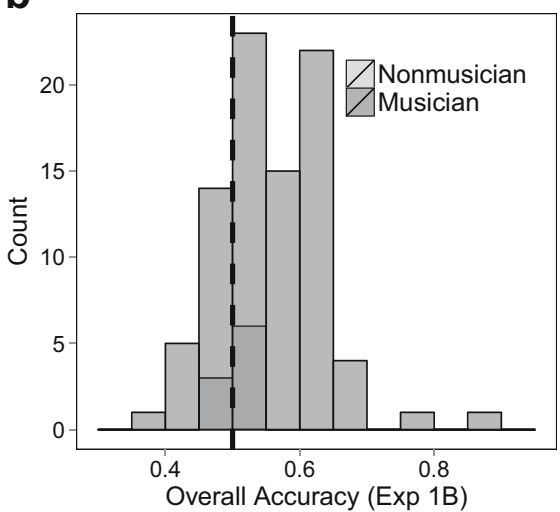

C

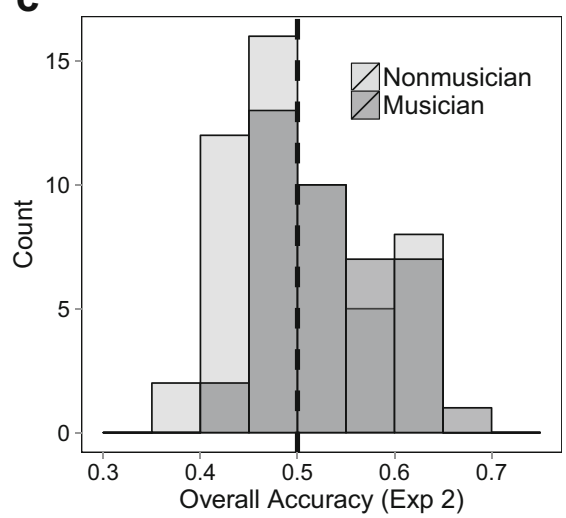

alter the interpretation of our results. The reported analyses include these highly accurate participants. The dotted vertical lines represent chance performance

\section{Results}

\section{Overall Intonation Accuracy}

Similar to Experiment 1, we found decisive evidence that participants could differentiate in-tune from out-of-tune notes. The proportion of times participants chose the correct intonation category was 0.550 (see Table 1 for comprehensive results). We were thus able to replicate the findings from Experiment 1 in a more controlled setting (i.e., while explicitly monitoring participants to ensure that they could not check their answers during the experiment). Figure $2 \mathrm{~b}$ shows a histogram of overall intonation accuracy across both musicians and nonmusicians.

\section{Discussion}

Experiments 1 and 1B provide evidence for a previously undocumented kind of absolute pitch processing - implicit $a b$ solute intonation (IAI). IAI allows an individual to label isolated notes as "in-tune" or "out-of-tune" without a tuning reference and without the ability to identify the note category. This ability suggests that the average listener has formed absolute categories for musical notes, even though he or she lacks the explicit ability to label these musical notes. Although genuine AP listeners may have greater precision in their category representations compared to non-AP possessors (Heald, Van Hedger, \& Nusbaum, 2014), this experiment demonstrates that these widespread implicit note categories preserve some graded distinctions in auditory pitch.

To tell when there is an intonation error, listeners in this experiment must have a sense of the standard against which each note is "in-tune" or "out-of-tune." In other words, they

must possess some implicit representation of prototypical note categories. This marks an important distinction between prior assessments of implicit absolute pitch ability, in which previous episodic experiences (e.g., a recording) can be recognized as matching or deviant from prior experience. Here, it is unlikely that the listener is accessing a particular episodic experience of an isolated note. This distinction is a potential explanation for the comparatively small effect in the present experiments.

\section{Experiment 2}

One unanswered question from Experiment 1 is whether IAI is instrument specific - that is, whether individuals can generalize beyond the instrumental timbres they commonly encounter and apply IAI to unfamiliar timbres. On the one hand, experience with detuned intonation in an AP population only influenced the intonation of subsequent notes for the same timbre (Hedger et al., 2013). This suggests that intonation perception may be grounded in particular instrumental listening experiences. On the other hand, it has been suggested that the process of implicitly acquiring information from statistical regularities and the application of this information to novel situations reflect the same general learning mechanism (e.g., Aslin \& Newport, 2012), which, in the present context, might allow an individual to correctly label any pitched sound with respect to canonical tuning. If IAI is based on the same kind of representation as used by AP listeners, albeit an unlabeled and implicit graded category structure for notes, we would predict that the results in Experiment 1 should be instrument specific. However, if IAI is a generic ability informed by experience, then perhaps the fundamental frequency of the stimulus is the only thing that matters in absolute intonation identification. 
Table 2 Experiment 2 intonation accuracy and statistical analyses. Accuracy is represented in terms of both the proportion correct and the number of total correct trials. We analyzed the data through a one-sample $t$ test as well as through a Bayesian equivalent of a one-sample $t$ test (represented through the Bayes Factor, $\mathrm{BF}_{10}$ ). The final column represents the $95 \%$ confidence interval of the standardized effect size

Experiment 2

\begin{tabular}{|c|c|c|c|c|c|c|}
\hline \multicolumn{7}{|c|}{ All participants $(n=93)$} \\
\hline Timbre & Mean Proportion Correct & Mean Number Correct & $t(d f)$ & $p$ value & $\mathrm{BF}_{10}$ & $95 \% \mathrm{CI}(\delta)$ \\
\hline Inv. Sine & 0.511 & $12.26 / 24$ & $1.07(92)$ & 0.286 & $1 / 5$ & {$[-0.09,0.31]$} \\
\hline Triangle & 0.515 & $12.36 / 24$ & $1.59(92)$ & 0.115 & $1 / 3$ & {$[-0.04,0.36]$} \\
\hline Overall & 0.513 & $24.62 / 48$ & $1.80(92)$ & 0.075 & $1 / 2$ & {$[-0.02,0.38]$} \\
\hline \multicolumn{7}{|c|}{ Musicians $(n=40)$} \\
\hline Timbre & Mean Proportion Correct & Mean Number Correct & $t(d f)$ & $p$ value & $\mathrm{BF}_{10}$ & $95 \% \mathrm{CI}(\delta)$ \\
\hline Inv. Sine & 0.542 & $13.01 / 24$ & $2.77(39)$ & 0.009 & 5 & {$[0.09,0.73]$} \\
\hline Triangle & 0.522 & $12.53 / 24$ & $1.35(39)$ & 0.185 & $1 / 3$ & {$[-0.10,0.51]$} \\
\hline Overall & 0.532 & $25.53 / 48$ & $3.00(39)$ & 0.005 & 8 & {$[0.13,0.77]$} \\
\hline \multicolumn{7}{|c|}{ Nonmusicians $(n=53)$} \\
\hline Timbre & Mean Proportion Correct & Mean Number Correct & $t(d f)$ & $p$ value & $\mathrm{BF}_{10}$ & $95 \%$ CI $(\delta)$ \\
\hline Inv. Sine & 0.487 & $11.70 / 24$ & $-1.00(52)$ & 0.322 & $1 / 4$ & {$[-0.39,0.13]$} \\
\hline Triangle & 0.510 & $12.24 / 24$ & $0.88(52)$ & 0.383 & $1 / 5$ & {$[-0.15,0.39]$} \\
\hline Overall & 0.499 & $23.94 / 48$ & $-0.13(52)$ & 0.901 & $1 / 7$ & {$[-0.28,0.24]$} \\
\hline \multicolumn{7}{|c|}{ Musicians vs. Nonmusicians (Difference Score) } \\
\hline Timbre & Mean Proportion Correct & Mean Number Correct & $t(d f)$ & $p$ value & $\mathrm{BF}_{10}$ & $95 \% \mathrm{CI}(\delta)$ \\
\hline Inv. Sine & 0.054 & $1.30 / 24$ & $2.78(91)$ & 0.007 & 6 & {$[0.12,0.95]$} \\
\hline Triangle & 0.012 & $0.03 / 24$ & $0.60(91)$ & 0.550 & $1 / 4$ & {$[-0.27,0.50]$} \\
\hline Overall & 0.033 & $1.58 / 48$ & $2.32(91)$ & 0.023 & 2 & {$[0.04,0.85]$} \\
\hline
\end{tabular}

\section{Method}

\section{Participants}

Ninety-four naïve participants were recruited through Amazon Mechanical Turk (MTurk). Participants had to be residing in the United States as well as have a minimum $90 \%$ satisfactory completion rate from prior MTurk assignments. Although we did not encourage musicians or nonmusicians to participate, roughly half of the participants $(n=41)$ reported at least some musical training. One participant was excluded from all analyses as they reported possessing AP. This left 93 analyzable participants (40 musicians, 53 nonmusicians).

\section{Materials and Procedure}

The materials were identical to those in Experiment 1 with the exception of the musical notes, which were generated in Adobe Audition (Adobe Systems; San Jose, CA) with either an inverted sine wave timbre ${ }^{3}(n=24)$ or a triangle wave timbre $(n=24)$. Similar to Experiment 1, we did not need to shift any

\footnotetext{
$\overline{3}$ The inverted sine wave signal option in Adobe Audition generates a complex waveform with nine harmonics and an approximate 11-dB drop between adjacent harmonics. Thus it is not a "pure" tone.
}

audio files in pitch, because we were able to generate the exact frequencies of both in-tune and out-of-tune notes. The frequency range was identical to that in Experiment 1 (24 notes in the range of $\mathrm{A}[3]=220 \mathrm{~Hz}$ to $\mathrm{G \#} \mathrm{[4]}{ }^{+50}=427.7 \mathrm{~Hz}$ ). Additionally, the experimental procedure remained the same. Table S1 summarizes the musical experience results from all participants.

\section{Results}

\section{Overall Intonation Accuracy}

Unlike Experiment 1, we did not find evidence that all participants were able to differentiate canonically tuned from noncanonically tuned notes when using unfamiliar timbres (see Table 2). The overall proportion of trials in which participants were able to differentiate canonically tuned from noncanonically tuned notes was 0.513 .

\section{Group Differences}

Similar to Experiment 1, we separately assessed whether musicians might show enhanced performance relative to nonmusicians. Our group of 40 analyzable musicians was able to correctly select the intonation category of isolated 
notes with a proportion of 0.532 . This proportion, while providing moderate support that musicians could differentiate canonically in-tune from out-of-tune notes, was significantly lower than the musician performance in Experiment $1, t(86)=3.21, p=.002, \mathrm{BF}_{10}=18$. Although musicians seemed to outperform nonmusicians, the evidence for this musician advantage was weak. Figure $2 \mathrm{c}$ shows a histogram of overall intonation accuracy across both musicians and nonmusicians.

\section{Discussion}

Experiment 2 demonstrates that IAI does not strongly generalize to unfamiliar timbres. Both musicians and nonmusicians displayed poorer performance for inverted sine wave and triangle wave notes compared to piano and violin notes (see Experiment 1). Collapsed across both groups, performance was not above chance. Moreover, although the musician group independently was above chance, practically speaking the effect was so small that it could be considered functionally equivalent to chance performance. This argues that the implicit note categories used by musicians and nonmusicians are based on familiarity derived from experience. Although the two timbres used are not frequently experienced, they are part of the repertoire of music synthesis (coming from a music synthesizer), and musicians are more likely to have some contact with these timbres. Overall, these results are reminiscent of the timbral specificity of AP possessors' note representations.

\section{General Discussion}

The present experiments demonstrate that individuals who do not possess absolute pitch are able to provide absolute intonation judgments, even in the context of listening to isolated musical notes. This suggests that non-AP possessors have pitch representations that include typicality, which was previously thought to be exclusive to individuals with AP (cf. Miyazaki, 1988). This more widespread effect, which we have labeled implicit absolute intonation (IAI), appears to be stronger in musicians compared to nonmusicians, though both groups were independently above chance when judging familiar instrumental timbres. When judging unfamiliar timbres, however, the performance of nonmusicians was no longer distinguishable from chance, and the performance of musicians was significantly lower than what was observed for familiar instrumental timbres. These findings suggest that IAI partly interacts with musical training and might not generalize to novel timbres.

Why would musical training improve IAI ability? One possibility is that the added sensorimotor experience gained by musicians may enhance absolute note representations (cf.
Cuddy, 1968; Lundin, 1963) or improve pitch discriminability (Kishon-Rabin, Amir, Vexler, \& Zaltz, 2001), consequently improving IAI. Additionally, it is possible that musical training does not cause better IAI. For instance, it is possible that some individuals have an inherently better ability to process pitch absolutely (e.g., see Ross, Olson, \& Gore, 2003), which then draws these individuals to musical instruction. However, perhaps the most parsimonious explanation is that musicians may possess greater experience listening to canonically in-tune notes compared to the general population. This additional familiarity with canonically tuned notes in some sense can be compared to familiarity with a given music recording, in which increased listening experience for a given popular song is known to lead to more accurate absolute pitch judgments (e.g., Schellenberg \& Trehub, 2003).

Another unresolved question is why IAI does not appear to generalize to unfamiliar timbres. Hedger et al. (2013) reported that brief experiences with altered intonation could temporarily shift an AP possessor's intonation, though this reorientation of intonation did not appear to generalize to novel timbres. Similarly, in the present experiments we found no overall evidence for generalization to unfamiliar timbres and weak evidence for generalization among musicians, though this "generalization" could have been actually due to limited experience with the unfamiliar timbres (computer-generated complex tones are not completely novel in musical settings). Overall, these results point to the notion that implicit absolute intonation knowledge might need to be independently built up within particular timbre categories. The lack of generalization to unfamiliar timbres fits within the emerging framework for musical note categorization, which has been extensively studied among AP possessors and suggests that note representations are not completely abstract but rather grounded by timbre (e.g., Bahr, Christensen, \& Bahr, 2005; Van Hedger, Heald, \& Nusbaum, 2015).

Overall, these results demonstrate that listeners are sensitive to fine-grained intonation information in their musical listening environments and can apply this knowledge of note category typicality to an artificial setting (i.e., making judgments about isolated musical notes). The ability to absolutely label an isolated note as "in-tune" or "out-of-tune"-previously thought to be exclusively within the realm of AP possessors - thus appears to be a more widespread ability. Furthermore, the consistent finding that musicians performed better than nonmusicians suggests that this implicit sense of absolute intonation can be sharpened through particular experiences.

Acknowledgments and Notes This research was supported by the Multidisciplinary University Research Initiatives (MURI) Program of the Office of Naval Research through Grant DOD/ONR N0001413-1-0205. The data for all experiments are uploaded to the Open Science Framework and can be accessed using the following URL: https://osf.io/vjtx3/. 


\section{References}

Aslin, R. N., \& Newport, E. L. (2012). Statistical learning: From acquiring specific items to forming general rules. Current Directions in Psychological Science, 21(3), 170-176. doi:10.1177 10963721412436806

Bahr, N., Christensen, C. A., \& Bahr, M. (2005). Diversity of accuracy profiles for absolute pitch recognition. Psychology of Music, 33(1), 58-93. doi:10.1177/0305735605048014

Ben-Haim, M. S., Eitan, Z., \& Chajut, E. (2014). Pitch memory and exposure effects. Journal of Experimental Psychology: Human Perception and Performance, 40(1), 24-32. doi:10.1037/a0033583

Cuddy, L. L. (1968). Practice effects in the absolute judgment of pitch. The Journal of the Acoustical Society of America, 43(5), 10691076. doi:10.1121/1.1910941

Darwin, C. J., Turvey, M. T., \& Crowder, R. G. (1972). An auditory analogue of the Sperling partial report procedure: Evidence for brief auditory storage. Cognitive Psychology, 3(2), 255-267. doi:10.1016 /0010-0285(72)90007-2

de Leeuw, J. R. (2014). jsPsych: A JavaScript library for creating behavioral experiments in a Web browser. Behavior Research Methods, 1-12. doi:10.3758/s13428-014-0458-y

Deutsch, D. (2013). Absolute pitch. In D. Deutsch (Ed.), The psychology of music (3rd ed., pp. 141-182). San Diego, CA: Academic Press. doi:10.1016/B978-0-12-381460-9.00005-5

Heald, S. L. M., Van Hedger, S. C., \& Nusbaum, H. C. (2014). Auditory category knowledge in experts and novices. Frontiers in Neuroscience, 8(260). doi:10.3389/fnins.2014.00260

Hedger, S. C., Heald, S. L. M., \& Nusbaum, H. C. (2013). Absolute pitch may not be so absolute. Psychological Science, 24(8), 1496-1502. doi:10.1177/0956797612473310

Jakubowski, K., \& Müllensiefen, D. (2013). The influence of musicelicited emotions and relative pitch on absolute pitch memory for familiar melodies. Quarterly Journal of Experimental Psychology, 66(7), 1259-1267. doi:10.1080/17470218.2013.803136
JASP Team. (2016). JASP (Version 0.7.5.6)[Computer software]. Retrieved from https://jasp-stats.org/faq/ (accessed 28 April 2016)

Kishon-Rabin, L., Amir, O., Vexler, Y., \& Zaltz, Y. (2001). Pitch discrimination: Are professional musicians better than non-musicians? Journal of Basic and Clinical Physiology and Pharmacology, 12(2), 125-143.

Levitin, D. J. (1994). Absolute memory for musical pitch: evidence from the production of learned melodies. Perception \& Psychophysics, 56(4), 414-423. doi:10.3758/BF03206733

Lockhead, G. R., \& Byrd, R. (1981). Practically perfect pitch. Journal of the Acoustical Society of America, 70(2), 387-389.

Lundin, R. W. (1963). Can perfect pitch be learned? Music Educators Journal, 49(5), 49-51.

Miyazaki, K. (1988). Musical pitch identification by absolute pitch possessors. Perception \& Psychophysics, 44(6), 501-512. doi:10.3758 /BF03207484

Rosch, E. H. (1973). Natural categories. Cognitive Psychology, 4(3), 328-350.

Ross, D. A., Olson, I. R., \& Gore, J. C. (2003). Absolute pitch does not depend on early musical training. Annals of the New York Academy of Sciences, 999, 522-526. doi: 10.1196/annals.1284.065

Saffran, J. R., Johnson, E. K., Aslin, R. N., \& Newport, E. L. (1999). Statistical learning of tone sequences by human infants and adults. Cognition, 70(1), 27-52. doi:10.1016/S0010-0277(98)00075-4

Schellenberg, E. G., \& Trehub, S. E. (2003). Good pitch memory is widespread. Psychological Science, 14(3), 262-266. doi:10.1111 /1467-9280.03432

Van Hedger, S. C., Heald, S. L. M., \& Nusbaum, H. C. (2015). The effects of acoustic variability on absolute pitch categorization: Evidence of contextual tuning. The Journal of the Acoustical Society of America, 138(1), 436-446. doi:10.1121/1.4922952

Ward, W. D., \& Burns, E. M. (1982). Absolute pitch. In D. Deutsch (Ed.), The psychology of music (pp. 431-451). San Diego, CA: Academic Press. 